\title{
GROUPS OF DIFFEOMORPHISMS AND THE SOLUTION OF THE CLASSICAL EULER EQUATIONS FOR A PERFECT FLUID
}

\author{
BY DAVID G. EBIN AND JERROLD E. MARSDEN
}

Communicated by Jürgen K. Moser, February 4, 1969

1. Introduction. We announce several results on the structure of the group of diffeomorphisms $D$ of a compact $n$-manifold $M$, possibly with boundary. The group $D$ has the structure of a differentiable manifold modelled on a Fréchet space and with this structure, the group operations are smooth. See Leslie [5] and Omori [8], for the proof in case $M$ has no boundary. Following Omori, we call $D$ an ILH Lie group.

We shall show that several infinite dimensional subgroups of $D$ are actually (ILH) submanifolds and hence also have the structure of ILH Lie groups. Also, we construct certain (weak) Riemannian structures on $D$ (and on certain subgroups) and find the geodesic flows associated to them.

More specifically, if $\mu$ is a smooth volume on $M$ and $D_{\mu}$ consists of all diffeomorphisms leaving $\mu$ invariant then $D_{\mu}$ is a closed submanifold of $D$. This group $D_{\mu}$ hence has an ILH Lie group structure. This group is of fundamental importance in the study of hydrodynamics of perfect fluids in $M$, as it is the appropriate configuration space. Arnold [1] has shown that the solution of the classical Euler equations for the fluid corresponds to geodesics on $D_{\mu}$ relative to a given invariant metric on $D_{\mu}$ induced by a Riemannian structure on $M$. (See Marsden and Abraham [6] for a precise proof of Arnold's theorem.) We prove the existence of such a smooth geodesic flow and hence that the Euler equations have unique smooth solutions, existing for short time, and varying smoothly with respect to the initial conditions. We expect that the flow is complete and thus the solutions can be uniquely extended for all time. Weaker versions of the above result were proven by Kato [4] and others for two and three dimensional regions in Euclidean space, although Kato [4] shows that in the two dimensional case, solutions do exist for all time.

We are indebted to many people for their remarks and suggestions; especially J. Dowling, T. Kato, I. Kupka, R. Palais and C. Robinson.

2. Technicalities. In order to prove our results we must enlarge the group $D$ slightly so that it becomes a Hilbert manifold. We define $D^{\circ}$ (called the set of $H^{*}$ diffeomorphisms) to be the set of all maps $\eta$ : 
$M \rightarrow M$ such that $\eta$ and $\eta^{-1}$ each have square integrable distribution derivatives up to order $s$. (Elements of $D^{s}$ are $C^{k}$ if $k<s-n / 2$ ). For $s>n / 2+1, D^{s}$ is a Hilbert manifold based on the Hilbert space $H^{s}(T)$ of $H^{*}$ vector fields of $M$, (see Corollary 1 below if $M$ has boundary); see Ebin [3] for details. Then $D^{s}$ is a topological group; right multiplication is a smooth map, but left multiplication and inversion are only continuous. (The same is true for the group of $C^{k}$ diffeomorphisms.) The group $D^{8}$ is similar to a Lie group in that:

Proposition. Let $s>n / 2+2$. Given a tangent vector $X$ at the identity of $D^{s}$ there exists a unique $C^{1}$ 1-parameter subgroup $\gamma_{X}(t)$ of $D^{s}$ tangent to $X$ at the identity. The resulting exponential map, $\left(\exp (X)=\gamma_{X}(1)\right)$ is continuous but not $C^{1}$.

OUTLINE OF PROOF. Showing the existence of $\gamma_{X}$ is the same as proving that the integral flow of an $H^{s}$ vector field consists of $H^{s}$ diffeomorphisms. This can be done by modifying the method used in Robbin [6] for the case of $C^{k}$ vector fields, and by analysing the linear nonhomogeneous equation which is satisfied by the second derivative of the flow. For the latter we are indebted to I. Kupka. The map "exp" cannot be $C^{1}$ because it does not cover a neighborhood of the identity.

3. Results. We shall assume $s>n / 2+1$.

THEOREM 1. Let $M$ be a compact without boundary and let $\omega$ be either a volume element or a symplectic two-form on $M$. Then $D_{\omega}^{s}$, the set of all $H^{s}$ diffeomorphisms leaving $\omega$ fixed, is a smooth closed submanifold of $D^{8}$. Also $\mathfrak{D}_{\omega}$ is a Fréchet submanifold of $\mathfrak{D}^{\text {, and }} \mathfrak{D}_{\omega}$ has the structure of an ILH Lie group.

OUTLINE OF PROOF. We consider the space of $H^{s-1} k$-forms, $H^{s-1}\left(\Lambda^{k}\right)$. Then $d\left(H^{s}\left(\Lambda^{k-1}\right)\right.$ ) is a closed subspace of $H^{s-1}\left(\Lambda^{k}\right)$, (using Hodge theory for example) and it is tangent to the affine space $[\omega]^{s-1}=\omega$ $+d\left(H^{s}\left(\Lambda^{k-1}\right)\right)$, where $k$ equals $n$ or 2 (depending on whether $\omega$ is a volume element or symplectic form). Let $\psi: D^{s} \rightarrow \mathrm{H}^{s-1}\left(\Lambda^{k}\right) ; \psi(\eta)=\eta^{*}(\omega)$, the usual pull back. By the methods of Ebin [3], we find that $\psi$ is smooth, and that $T_{e}(\psi): H^{s}(T) \rightarrow H^{s-1}\left(\Lambda^{k}\right)$ the tangent map of $\psi$ at the identity $e$, is: $X \mapsto d(i(X) \omega)$. This map is onto $d\left(H^{s}\left(\Lambda^{k-1}\right)\right)$, so near $e, \psi$ is a submersion of $D^{s}$ onto $[\omega]^{s-1}$. Using right multiplication in $D^{s}$ we find that $\psi$ is a submersion everywhere on an open set containing $D_{\omega}^{s}$ so $\psi^{-1}(e)=D_{\omega}^{s}$ is a submanifold.

THEOREM 2. Let $M$ be as above and $N$ a closed submanifold. Let $\mathscr{D}_{N}^{s}=\left\{\eta \in D^{8} \mid \eta(N) \subset N\right\}$ and $\mathscr{D}_{N, p}^{s}=\left\{\eta \in D^{s} \mid \forall x \in N, \eta(x)=x\right\}$. Then 
$\mathscr{D}_{N}^{s}$ and $\mathscr{D}_{N, p}^{s}$ are closed submanifolds of $\mathscr{D}^{*}$, and the same is true when " $s$ " is removed from each set. These groups are also ILH Lie groups. The tangent spaces at e, $H_{N}^{s}, H_{N, p}^{s}$ are specified below.

OUTLINE OF PROOF. Using a tubular neighborhood, find a Riemannian structure on $M$ such that $N$ is a totally geodesic submanifold. If exp: $T(M) \rightarrow M$ is the corresponding exponential map, $\Omega_{\mathrm{xp}}: H^{*}(T)$ $\rightarrow D^{s}$ defined by $X \mapsto \exp \circ X$ is a natural chart for $\mathbb{D}^{8}$, defined in a neighborhood of zero in $H^{s}(T)$. Let

$$
H_{N}^{*}(T)=\left\{X \in H^{s}(T) \mid X \uparrow N: N \rightarrow T(N)\right\}
$$

and $H_{N, p}^{s}(T)=\left\{X \in H^{s}(T) \mid X \uparrow N=0\right.$-section $\}$, $\uparrow$ denoting restriction. Since $N$ is totally geodesic, $\Omega_{\exp }\left\lceil H_{N}^{s}(T)\right.$ and $\Omega_{\exp }\left\lceil H_{N, p}^{s}(T)\right.$ define submanifold charts about the identity of $D_{N}^{s}$ and $D_{N, p}^{s}$ respectively. Using right translation we get charts everywhere, and the theorem follows.

CoRollary 1. Let $(M, \partial M)$ be a compact manifold with boundary, and $D^{s}$ its group of $\mathrm{H}^{*}$ diffeomorphisms. Let $\mathfrak{D}_{p}^{s}$ be the subgroup leaving the boundary pointwise fixed. Then $D^{\circ}$ is a Hilbert manifold and topological group as in the nonboundary case (so for example, right multiplication is smooth), and $D_{p}^{s}$ is a closed submanifold. The corresponding result holds with " $s$ " removed.

OutLine of PROoF. Consider $\hat{M}$, the double of $(M, \partial M)$, and the group $\mathscr{D}_{\partial M}^{s}(\hat{M})$ of $H^{s}$ diffeomorphisms of $\hat{M}$ leaving $\partial M$ invariant. By restricting this group to its action on $M$, we get the corollary.

CoRollary 2. Let $M$ have boundary and let $\omega$ be a volume element. Then $D_{\omega}^{s}$ and $D_{p, \omega}^{s}$ the subgroups of $D^{s}$ and $D_{p}^{s}$ leaving $\omega$ fixed, are closed submanifolds of $D^{s}$. If $\omega$ is an exact symplectic 2 -form, $\mathfrak{D}_{p, \omega}^{s}$ is again a submanifold.

METHOD OF PROOF. We use the method of Theorem 1, considering the de Rham cohomology and Hodge theory for manifolds with boundary and following the work of Duff and Spencer [2].

REMARK. Using the proof of Theorem 1, the methods in Moser [7], and the above corollary one can prove a theorem of Omori [8] that $D_{\omega}$ is a deformation retract of $D$. Our proof is simpler than Omori's in that it does not use the heat equation. It extends the theorem to the boundary case, and also shows that the deformation is smooth.

In the sequel, $M$ will be a compact Riemannian manifold without boundary with Riemannian metric $\langle$,$\rangle . Let \nabla$, exp, and $\mu$ be the corresponding connection, exponential map, and volume element 
respectively. We define a weak Riemannian structure $($,$) on D^{\circ}$ as follows: Let $X$ and $Y$ be tangent vectors and $\eta \in D^{s}$. They are $H^{*}$ maps from $M$ into $T(M)$ which cover $\eta$; i.e. the following diagram commutes:

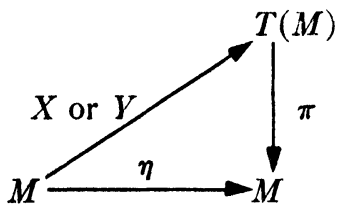

Define $(X, Y)=\int_{M}\langle X, Y\rangle_{\mu}$ which can be written $\int_{M}\left\langle X_{p}, Y_{p}\right\rangle_{\eta(p)} \mu(p)$, as $p$ ranges over $M$.

THEOREM 3. (, ) is invariant under right multiplication by $\mathfrak{D}_{\mu}^{s}$. It induces a smooth connection $\bar{\nabla}$ and exponential map $E$ on $D^{s}$ which are also right invariant under $\mathfrak{D}_{\mu}^{s} . E(X)=\exp \circ X$, and the natural charts discussed in the proof of Theorem 2 provide normal neighborhoods about each point.

OUTLINE OF PROOF. Let $R_{\eta}: D^{s} \rightarrow D^{8}$ be right multiplication and TR its tangent map. Then $\mathrm{TR}_{\eta}(X)=X \circ \eta$, so if $\eta \in \mathcal{D}_{\mu}^{s}$, $\left(\mathrm{TR}_{\eta} \cdot X, \mathrm{TR}_{\eta} \cdot Y\right)$ $=\int_{M}\langle X \circ \eta, Y \circ \eta\rangle \mu=\int_{M}\langle X, Y\rangle_{\eta} *(\mu)=(X, Y)$. For the rest of the theorem we need only show that the natural charts are smooth normal charts at each point. That is, given $X, Y, Z$ tangent vectors at $\eta \in D^{\circ}$ we extend them locally to vector fields $\hat{X}=T\left(\Omega_{\exp }\right)(X)$, $\hat{Y}=T\left(\Omega_{\exp }\right)(Y), \hat{Z}=T\left(\Omega_{\text {exp }}\right)(Z)$, about $\eta$ where $\Omega_{\text {exp }}: T\left(D^{s}\right) \rightarrow D^{s}$ is defined by $X \mapsto \exp \circ X$ and $T\left(\Omega_{\exp }\right)$ is its tangent map. Then we show $\hat{X}(\hat{Y}, \hat{Z})_{\eta}=0$. But

$$
\begin{aligned}
\hat{X}(\hat{Y}, \hat{Z})_{\eta} & =\frac{d}{d t}\left[D \exp _{t X}(Y), D \exp _{t X}(Z)\right]_{t=0} \\
& =\left.\int_{M} \frac{d}{d t}\left\langle D \exp _{t X} Y, D \exp _{t X} Z\right\rangle_{\eta \circ \exp (t X)} \mu\right|_{t=0}=0
\end{aligned}
$$

because exp gives a normal coordinate chart at each point of $M$.

THEOREM 4. $D_{\mu}^{s}$ has a right invariant weak Riemannian structure as a submanifold of $D^{*}$. This structure gives it a smooth connection and exponential map.

OUTLINE OF PROOF. The structure is right invariant because $(,$, is right invariant under $\mathscr{D}_{\mu}^{s}$. Let P: $T\left(D^{*}\right)\left\lceil D_{\mu}^{s} \rightarrow T\left(D_{\mu}^{s}\right)\right.$ be the orthogonal projection defined by $($,$) . Then the connection of D_{\mu}^{s}$ is $P \cdot \bar{\nabla}$. To show it is smooth we must show $P$ is smooth. The theorem then 
will follow easily. On the tangent space at $e, P(X)=X-d \Delta^{-1} \delta X$ where $\delta$ is the divergence with respect to $\mu$, and $\Delta$ is the de RhamLaplace operator. Hodge theory tells us that the image of $P$ is closed. Also on the tangent space at $\eta, P_{\eta}=\mathrm{TR}_{\eta} \circ P_{e} \circ \mathrm{TR}_{\eta}^{-1}$. That this is smooth in $\eta$ follows from an argument like that of Ebin [3, Theorem 7.1].

CoRollary. The exponential map $E$ of $\mathfrak{D}_{\mu}^{s}$ restricts to an exponential map for $\mathfrak{D}_{\mu}$.

OUtLINE OF PROOF. Using an argument like [3, Theorem 7.5], we find that if $\eta \in D_{\mu}^{s+k}$, then $E(\eta) \in D_{\mu}^{s+k}$.

REMARK. Theorem 4 and its corollary remain true when $M$ has boundary. However the proof is more complicated again requiring Hodge theory for manifolds with boundary, as in Corollary 2 of Theorem 2.

4. Euler equations. The Euler equations on a compact Riemannian manifold possibility with boundary are the nonlinear equations of evolution given by

$$
\begin{aligned}
d \tilde{v} / d t+L_{v} \tilde{v} & =d g ;\left[L_{v}=\text { Lie derivative }\right] ;-\epsilon<t<\epsilon, \\
\operatorname{div} v & =0 ; v \| \text { boundary }(M)=\partial M, \\
\left.v\right|_{t=0} & =v_{0} ;\left[\operatorname{div} v_{0}=0, v_{0} \| \partial(M)\right],
\end{aligned}
$$

where $\tilde{v}$ is the one form obtained from the vectorfield $v$ (via the Riemannian metric), and $g$ is a function to be found ( $g$ is related to the pressure by $p=g+\langle v, v\rangle / 2)$. The flow $\sigma_{v_{0}}(t)$ of the time dependent vector field $v$ is the actual fluid motion and is the geodesic starting at $e$ in the above corollary. Theorem 9 of [6] together with the above corollary, shows that these equations are soluble for short time $t$; if $v_{0}$ is $C^{\infty}$, so is $v$. Note that for the geodesics $\sigma_{v_{0}}(t)$, if $v_{0}$ is $H^{s}$, then $\sigma_{\nu_{0}}(t)$ is an $H^{s}$ diffeomorphism and $v$ is $H^{s}$; note that left multiplication "loses derivatives," (whereas right multiplication does not). In the $C^{\infty}$ topology the solution $v$ varies differentiably $\left(C^{\infty}\right)$ with the initial (or Cauchy) data $v_{0}$. There is no similar theorem for $v=0$ on $\partial M$. The geodesics minimize the energy although this situation is not covered by the calculus of variations as developed by Palais and Smale (see [10]). Also, although the exponential maps of the Lie groups $D, D_{\omega}$ do not cover a neighborhood of the identity, the exponential maps associated with the metrics in Corollary 2 do.

Details of this, the rest of the above work, and some results on completeness will appear in a forthcoming paper. 
NOTE ADDED IN PROOF. The above results can also be used to study the Navier-Stokes equations in case $\partial M=\phi$. Using the Trotter product formula, one obtains existence, uniqueness and (strong) $H^{8}$ convergence of solutions as the viscosity $\nu \rightarrow 0$. (Existence is for a time independent of $\nu$.) Details are given in the above mentioned paper. The Euler equations are more commonly written

$$
d v / d t+\nabla_{v} v=d p \quad[\nabla=\text { covariant derivative }]
$$

and the Navier-Stokes equations become

$$
d v / d t-v \Delta v+\nabla_{v} v=d p \quad[\nabla=\text { Laplacian }] .
$$

One can also allow a force term $f$ on the right-hand sides.

\section{REFERENCES}

1. V. Arnol'd, Sur la gêométrie différentielle des groupes de Lie de dimension infinie et ses applications à l'hydrodynamique des fluides parfaits, Ann. Inst. Fourier (Grenoble) 16 (1966), 319-361.

2. G. Duff and D. Spencer, Harmonic tensors on Riemannian manifolds with boundary, Ann. of Math. (2) 56 (1952), 128-156.

3. D. Ebin, The manifold of Riemannian metrics, Proc. Sympos. Pure Math., vol. 15, Amer. Math. Soc., Providence, R.I. (to appear). (See also Bull. Amer. Math. Soc. 74 (1968), 1001-1003.)

4. T. Kato, On classical solutions of the two dimensional non-stationary Euler equation, Arch. Rational Mech. Anal. 25 (1967), 188-200.

5. J. Leslie, On a differential structure for the group of diffeomorphisms, Topology 6 (1967), 263-271.

6. J. Marsden and R. Abraham, Hamiltonian mechanics on Lie groups and hydrodynamics, Proc. Sympos. Pure Math., vol. 16, Amer. Math. Soc., Providence, R.I. (to appear).

7. J. Moser, On the volume elements on a manifold, Trans. Amer. Math. Soc. 120 (1965), 286-294.

8. H. Omori, On the group of diffeomorphisms on a compact manifold, Proc. Sympos. Pure Math., vol. 15, Amer. Math. Soc., Providence, R.I. (to appear).

9. J. Robbin, On the existence theorem for differential equations, Proc. Amer. Math. Soc. 19 (1968), 1005-1006.

10. S. Smale, Morse theory and a non-linear generalization of the Dirichlet problem, Ann. of Math. (2) 80 (1964), 382-396.

University of California, Berkeley, California 94720 